\title{
The insulin resistance phenotype (muscle or liver) interacts with the type of diet to determine changes in disposition index after 2 years of intervention: the CORDIOPREV-DIAB randomised clinical trial
}

\author{
Ruth Blanco-Rojo ${ }^{1,2,3,4}$ • Juan F. Alcala-Diaz ${ }^{1,2,3,4} \cdot$ Suzan Wopereis $^{5}$ • \\ Pablo Perez-Martinez ${ }^{1,2,3,4}$ - Gracia M. Quintana-Navarro ${ }^{1,2,3,4}$ - Carmen Marin ${ }^{1,2,3,4}$. \\ Jose M. Ordovas ${ }^{6,7}$ • Ben van Ommen ${ }^{5}$ - Francisco Perez-Jimenez ${ }^{1,2,3,4}$. \\ Javier Delgado-Lista ${ }^{1,2,3,4}$ • Jose Lopez-Miranda ${ }^{1,2,3,4}$
}

Received: 28 July 2015 / Accepted: 8 September 2015 / Published online: 16 October 2015

(C) Springer-Verlag Berlin Heidelberg 2015

\begin{abstract}
Aims/hypothesis The aim of the study was to determine whether basal insulin resistance (IR) phenotype (muscle and/ or liver) determines the effect of long-term consumption of a Mediterranean diet or a low-fat diet on tissue-specific IR and beta cell function.

Methods The study was performed in 642 patients included in The effect of an olive oil rich Mediterranean diet on type 2
\end{abstract}

Ruth Blanco-Rojo and Juan F. Alcala-Diaz contributed equally to this study.

Javier Delgado-Lista and Jose Lopez-Miranda are joint senior authors.

Electronic supplementary material The online version of this article (doi:10.1007/s00125-015-3776-4) contains peer-reviewed but unedited supplementary material, which is available to authorised users.

Jose Lopez-Miranda

jlopezmir@uco.es

1 Lipids and Atherosclerosis Research Unit, Reina Sofia University Hospital, Avda Menendez Pidal, s/n, 14004 Cordoba, Spain

2 Nutrigenomics and Metabolic Syndrome, Maimonides Institute for Biomedical Research at Cordoba (IMIBIC), Cordoba, Spain

3 Department of Medicine, University of Cordoba, Cordoba, Spain

4 CIBER Fisiopatología Obesidad y Nutrición (CIBEROBN), http://www.ciberobn.es/

5 TNO, Zeist, the Netherlands

6 Nutrition and Genomics Laboratory, Jean Mayer US Department of Agriculture Human Nutrition Research Center on Aging, Tufts University, Boston, MA, USA

7 IMDEA Food Institute, Madrid, Spain diabetes mellitus risk and incidence study (CORDIOPREVDIAB). A total of 327 patients were randomised to a Mediterranean diet (35\% fat; $22 \%$ from monounsaturated fatty acids) and 315 to a low-fat diet ( $<28 \%$ fat). At baseline, the patients were classified into four phenotypes according to the type of IR: (1) no IR; (2) muscle IR; (3) liver IR; (4) muscle+ liver IR. The hepatic insulin resistance index (HIRI), muscular insulin sensitivity index (MISI) and disposition index were analysed at baseline and after 2 years of follow-up.

Results At baseline, 322 patients presented no IR, 106 presented muscle IR, 109 presented liver IR, and 105 presented muscle+liver IR. With both dietary interventions, HIRI decreased in all patients $(p<0.001)$ and MISI increased in muscle IR and muscle+liver IR patients $(p<0.01)$. Long-term intake of the Mediterranean diet increased the disposition index and insulinogenic index in the muscle IR patients ( $p=0.042$ and $p=0.044$, respectively) and the disposition index in the muscle+liver IR patients $(p=0.048)$, whereas the low-fat diet increased the disposition index in the liver IR patients $(p=0.017)$. Conclusions/interpretation Although both diets improve insulin sensitivity, there are differences based on basal IR phenotypes. Moreover, according to insulinogenic and disposition index data, a low-fat diet might be more beneficial to patients with liver IR, whereas patients with muscle IR and muscle+liver IR might benefit more from a Mediterranean diet.

Trial registration ClinicalTrials.gov NCT00924937

Funding The study was supported by the Ministerio de Economia y Competitividad (AGL2012/39615) and by the Ministerio de Ciencia e Innovacion (PIE14/00005 and PI13/ 00023) 
Keywords Beta cell function · Dietary intervention $\cdot$ Insulin resistance $\cdot$ Low-fat diet $\cdot$ Mediterranean diet

$\begin{array}{ll}\begin{array}{l}\text { Abbreviations } \\ \text { CHD }\end{array} & \begin{array}{l}\text { Coronary heart disease } \\ \text { CORDIOPREV } \\ \text { Coronary Diet Intervention with Olive Oil } \\ \text { and Cardiovascular Prevention }\end{array} \\ \text { CORDIOPREV- } & \begin{array}{l}\text { The effect of an olive oil rich Mediterra- } \\ \text { nean diet on type } 2 \text { diabetes mellitus risk } \\ \text { and incidence study }\end{array} \\ \text { DIAB } & \text { C-reactive protein } \\ \text { CRP } & \text { Hepatic insulin resistance index } \\ \text { HIRI } & \text { Impaired fasting glucose } \\ \text { IFG } & \text { Insulinogenic index } \\ \text { IGI } & \text { Impaired glucose tolerance } \\ \text { IGT } & \text { Insulin resistance } \\ \text { IR } & \text { Insulin sensitivity index } \\ \text { ISI } & \text { Muscular insulin sensitivity index } \\ \text { MISI } & \end{array}$

\section{Introduction}

Cardiovascular disease and type 2 diabetes are currently considered the main causes of disability and death worldwide [1]. Both are complex metabolic disorders, and their simultaneous presence considerably increases the risk of macrovascular complications and death. In diabetic patients with a previous heart attack, the 7 year incidence of subsequent myocardial infarction is more than double that of non-diabetic individuals with previous myocardial infarction. Similarly, the recurrence rate of major atherosclerotic complications in type 2 diabetic patients with a prior cardiovascular event is very high: around $6 \%$ per year [2]. Therefore, prevention of type 2 diabetes should be a priority in cardiovascular patients.

Insulin resistance (IR) reflects defective insulin action predominantly in skeletal muscle and liver, which are the main insulin-sensitive target tissues [3]. As a consequence, insulin secretion increases to maintain normoglycaemia, a situation that could compromise pancreatic beta cell function and result in type 2 diabetes [4]. There is compelling clinical trial evidence that type 2 diabetes can be prevented or delayed by lifestyle interventions: among these, modification of the diet has been established as one of the most important contributing factors [5]. Two dietary approaches have been suggested as good alternatives to delay the onset of type 2 diabetes: first, the Mediterranean diet, rich in olive oil, seems to provide cardiovascular benefits and increase insulin sensitivity; it has been recently linked to lower type 2 diabetes incidence $[6,7]$. The other alternative is a low-fat diet, with a content of $<30 \%$ energy from total fat, which has been demonstrated to prevent type 2 diabetes [5] and has been recommended by the ADA [8].
Although the development of type 2 diabetes is tightly related to IR, it is unclear whether IR develops simultaneously in multiple organs and whether its severity varies depending on the organ. Moreover, there is evidence that interventions that increase insulin sensitivity are organ specific $[9,10]$. However, to the best of our knowledge, no long-term comparisons of different dietary patterns in individuals at risk of diabetes that take into account the extent of IR in target organs have been reported.

The present work aims to determine whether basal IR phenotype (muscle and/or liver) determines the effect of longterm consumption of a Mediterranean diet or a low-fat diet on tissue-specific IR and beta cell function.

\section{Methods}

Participants The Coronary Diet Intervention with Olive Oil and Cardiovascular Prevention (CORDIOPREV) study is an ongoing prospective, randomised, open, controlled trial of 1,002 patients receiving conventional treatment for coronary heart disease (CHD) who had their last coronary event more than 6 months before enrolment in one of two different dietary models (a Mediterranean diet and a low-fat diet) over a period of 5 years. The patients were recruited between November 2009 and February 2012, mostly at the Reina Sofia University Hospital, Cordoba, Spain. The eligibility criteria, design and methods of the CORDIOPREV clinical trial have been reported elsewhere $[11,12]$, and the protocol is registered at ClinicalTrials.gov (registration no. NCT00924937). The outcome investigated in this study relates to the one of the secondary outcomes of the CORDIOPREV study: metabolic control of carbohydrates (CORDIOPREV-DIAB).

Patients who had not had a diabetes diagnosis and/or were not receiving glucose-lowering treatment before the beginning of the study underwent an OGTT as part of the protocol of the CORDIOPREV-DIAB study. Patients were asked about their medical diagnosis and glucose-lowering treatment in an interview with an internal medicine physician and their response was confirmed by electronic medical records, which are available for all patients in Andalucía, Spain. According to the baseline OGTT, 242 patients had normal glucose tolerance (fasting glucose $<5.5 \mathrm{mmol} / 1,2 \mathrm{~h}$ glucose $<7.7 \mathrm{mmol} / 1, \mathrm{HbA}_{1 \mathrm{c}}$ $<47.5 \mathrm{mmol} / \mathrm{mol}[6.5 \%]$ ); 77 had impaired fasting glucose (IFG; fasting glucose 5.6-6.9 mmol/1), 80 had impaired glucose tolerance (IGT; 2 h glucose 7.8-11.0 mmol/l); 53 had both IFG and IGT; and 190 had type 2 diabetes (fasting glucose $\geq 7 \mathrm{mmol} / 1,2 \mathrm{~h}$ glucose $\geq 11.1 \mathrm{mmol} / 1$ or $\mathrm{HbA}_{1 \mathrm{c}} \geq 47.5 \mathrm{mmol} /$ mol $[6.5 \%])$. Once in the study, patients who received a biochemical diagnosis of diabetes, but who were not put on pharmacological treatment by their physicians, continued in the study. Patients who initiated pharmacological treatment for diabetes were excluded from the study. 
A total of 642 patients were randomised to two dietary treatment groups: 327 to a Mediterranean diet group and 315 to a low-fat diet group. There were no significant differences in the demographic and metabolic characteristics between the groups (see electronic supplementary material [ESM] Table 1). Patients lost before follow-up at 2 years are detailed in ESM Fig. 1.

The local ethics committees approved the trial protocol and its amendments, which follow the Declaration of Helsinki and good clinical practice. The experimental protocol conforms to international ethical standards [13]. Written informed consent was obtained from all study participants.

Study diets The low-fat diet comprised $<28 \%$ of energy from fat (12\% monounsaturated, $8 \%$ polyunsaturated and $8 \%$ saturated fatty acids), $57 \%$ from carbohydrates and $15 \%$ from proteins. The Mediterranean diet comprised 35\% of energy from fat $(22 \%$ monounsaturated, $6 \%$ polyunsaturated and $7 \%$ saturated fatty acids), $50 \%$ from carbohydrates and $15 \%$ from proteins. To ensure that the main fat source of the Mediterranean diet (extra-virgin olive oil) was identical for all patients in this group, the olive oil was given to the participants by the research team. Food packs, including low-fat foods (cereals, legumes, pasta, etc.) were provided for the patients randomised to the low-fat diet group.

\section{Dietary assessment and anthropometric measurements} The CORDIOPREV study was supervised by a team of registered dietitians. At the beginning of the study and every year, the dietitian had a face-to-face interview with each patient, during which was carried out a 137-item semi-quantitative food frequency questionnaire, validated in Spain [14], and a validated 14-item questionnaire of adherence to the Mediterranean diet to determine a Mediterranean diet score [15]. Participants in each intervention group received the same intensive dietary counselling. The dietitian adapted the participants' customary diet to the Mediterranean diet or to the low-fat diet, focusing on the overall daily diet rather than on isolated nutrients. Weight, height and waist circumference were also measured at baseline and again each year according to standardised protocols.

Biochemical measurements Venous blood for analysis of the participants' biochemical variables was collected in tubes containing EDTA after a $12 \mathrm{~h}$ overnight fast at baseline and after 2 years of follow-up. Lipid variables, serum insulin, plasma glucose and plasma concentration of $\mathrm{C}$-reactive protein (CRP) were determined as previously reported $[11,12]$.

\section{Estimation of IR, insulin secretion and beta cell function} indices Patients underwent a standard OGTT at baseline and after 2 years of follow-up. After an overnight fast, blood was sampled from a vein before oral glucose intake $(0 \mathrm{~min})$ and again after a $75 \mathrm{~g}$ flavoured glucose load (Trutol 75; Custom Laboratories, Baltimore, MD, USA). Blood samples were taken at 30, 60, 90 and 120 min to determine glucose and insulin concentrations [16].

The following indices were then estimated. The indices used to determine tissue-specific IR were: the hepatic insulin resistance index (HIRI) and the muscle insulin sensitivity index (MISI). HIRI was estimated by the validated method of Matsuda and DeFronzo [16], expressed as fasting insulin $(\mathrm{pmol} / \mathrm{l}) \times$ fasting glucose $(\mathrm{mmol} / \mathrm{l})$. MISI was measured according to the method of Abdul-Ghani et al [17]: MISI $=(\mathrm{dG} / \mathrm{dt}) /$ mean plasma insulin concentration, where $\mathrm{dG} / \mathrm{dt}$ is the rate of decay of plasma glucose concentration from its peak value to its nadir during the OGTT. Other IR indices determined were: insulin sensitivity index (ISI) $=$ $10,000 / \sqrt{ }$ [(fasting insulin [pmol/1] $\times$ fasting glucose $[\mathrm{mmol} / \mathrm{l}]) \times($ mean OGTT insulin $[\mathrm{pmol} / \mathrm{l}]) \times($ mean OGTT glucose [mmol/1])] [16]; and HOMA-IR [18]. Insulin secretion was measured by the insulinogenic index (IGI): $\mathrm{IGI}=[30 \mathrm{~min}$ insulin - fasting insulin $(\mathrm{pmol} / \mathrm{l})] /[30 \mathrm{~min}$ glucose-fasting glucose (mmol/l)] [19]. Finally, beta cell function was estimated by calculating the disposition index as follows: disposition index $=\mathrm{ISI} \times\left[\mathrm{AUC}_{30} \mathrm{~min}\right.$ insulin $/$ $\mathrm{AUC}_{30}$ min glucose], where $\mathrm{AUC}_{30}$ min is the area under the curve between baseline and $30 \mathrm{~min}$ of the OGTT for insulin (pmol/l) and glucose (mmol/l) measurements, respectively, calculated by the trapezoidal method [20].

Determination of muscle and liver IR groups At baseline, the patients were distributed into four groups according to the presence or absence of muscle and/or liver IR. For this purpose, we used a method based on that described by Abdul-Ghani et al [21]. The patients were divided into tertiles according to HIRI and MISI. The highest tertile of HIRI and the lowest tertile of MISI were considered to indicate IR in each organ. A second operational definition based on the median value for IR in skeletal muscle and liver gave similar results.

Statistical analyses Normal distribution was tested for all measured variables, and skewed variables were normalised by $\log _{10}$. Statistical analysis was carried out using SPSS software version 18.0 for Windows (SPSS, Chicago, IL, USA). The data were presented as mean $\pm \mathrm{SD}$ for continuous variables and as frequencies for categorical variables. In the statistical tests, age, sex, BMI and change in weight were included as covariates. The statistical significance of the differences in mean values at baseline according to IR groups was assessed by a univariate general linear model. A repeated measures ANOVA test was used to determine the statistical differences between variables at baseline and after 2 years of follow-up. The mean percentage change in each variable was calculated as follows: $\left(\left[V_{2}-V_{0}\right] / V_{0}\right) \times 100$, where $V_{0}$ is the value of each variable at baseline and $V_{2}$ is the value after 
2 years of follow-up. A negative value of mean percentage change indicates that the variable has decreased after 2 years of follow-up, and a positive value indicates that the variable has increased. Bonferroni's test was used in all cases where post hoc analyses were required, in which $p<0.05$ was considered to be significant.

\section{Results}

Study population Table 1 shows the demographic and metabolic characteristics at baseline of the patients according to the basal IR phenotype. A group of 322 patients presented no muscle or liver IR, 106 had muscle IR, 109 had liver IR, and 105 presented both muscle and liver IR.

Patients with muscle IR, liver IR and muscle+liver IR showed significant differences compared with those with no IR in several variables. Patients with muscle IR were significantly older than those in the other groups $(p<0.001)$. As regards anthropometric measurements, patients with liver IR and muscle+liver IR showed significantly greater weight, BMI and waist circumference than those with muscle IR and those with no IR $(p<0.001)$. Serum triacylglycerol values were higher in patients with liver IR and muscle+liver IR than in those with no IR $(p<0.001)$. HDL-cholesterol was lowest in patients with liver IR and muscle+liver IR compared with the other groups $(p=0.017)$. Patients with liver IR and muscle + liver IR showed significantly greater $\mathrm{HbA}_{1 \mathrm{c}}$, fasting glucose and fasting insulin than those with muscle IR and those with no IR $(p<0.001)$.

With respect to the OGTT-derived indices (Table 1), HIRI was highest in patients with muscle+liver IR, and progressively decreased in the liver IR, muscle IR and no IR groups $(p<0.001)$. ISI was lowest, however, in patients with muscle+ liver IR, and progressively increased in the liver IR, muscle IR and no IR groups $(p<0.001)$. MISI was lower in patients with muscle+liver IR and muscle IR compared with those with liver IR and no IR $(p<0.001)$, whereas IGI was higher in patients with muscle+liver IR and muscle IR compared with those with liver IR and no IR $(p<0.001)$. The muscle+liver IR

Table 1 Demographic and metabolic characteristics of the patients by IR phenotype

\begin{tabular}{|c|c|c|c|c|c|}
\hline Characteristic & No IR & Muscle IR & Liver IR & Muscle+liver IR & $p$ value $^{\mathrm{a}}$ \\
\hline$n$ & 322 & 106 & 109 & 105 & \\
\hline Men/women $(n)$ & $275 / 47$ & $85 / 21$ & $96 / 13$ & $87 / 18$ & NS \\
\hline Age (years) & $58.49 \pm 9.43$ & $61.35 \pm 9.00 * \nsubseteq \S$ & $56.30 \pm 9.40$ & $57.04 \pm 8.68$ & $<0.001$ \\
\hline Weight (kg) & $80.35 \pm 12.23$ & $79.10 \pm 12.03$ & $91.51 \pm 14.04^{* \dagger}$ & $89.45 \pm 14.17 *^{\dagger}$ & $<0.001$ \\
\hline BMI $\left(\mathrm{kg} / \mathrm{m}^{2}\right)$ & $29.30 \pm 3.95$ & $29.62 \pm 3.66$ & $32.28 \pm 4.28^{* \dagger}$ & $32.67 \pm 4.71 *^{\dagger \dagger}$ & $<0.001$ \\
\hline Waist circumference (cm) & $100.4 \pm 10.1$ & $100.2 \pm 10.3$ & $109.3 \pm 9.6^{* \dagger}$ & $108.0 \pm 11.1^{* \dagger}$ & $<0.001$ \\
\hline Serum triacylglycerols $(\mathrm{mmol} / \mathrm{l})$ & $1.30 \pm 0.69$ & $1.34 \pm 0.64$ & $1.62 \pm 0.71^{*}$ & $1.74 \pm 0.75^{* \dagger}$ & $<0.001$ \\
\hline Total cholesterol (mmol/l) & $4.17 \pm 0.80$ & $4.16 \pm 0.80$ & $4.15 \pm 0.79$ & $4.31 \pm 0.85$ & NS \\
\hline HDL-cholesterol (mmol/l) & $1.16 \pm 0.25$ & $1.11 \pm 0.22$ & $1.06 \pm 0.21^{* \dagger}$ & $1.06 \pm 0.23^{* \dagger}$ & 0.017 \\
\hline LDL-cholesterol (mmol/l) & $2.38 \pm 0.66$ & $2.41 \pm 0.66$ & $2.26 \pm 0.59$ & $2.42 \pm 0.67$ & NS \\
\hline $\mathrm{CRP}(\mathrm{nmol} / \mathrm{l})$ & $20.29 \pm 19.62$ & $21.43 \pm 18.38$ & $23.81 \pm 17.33$ & $22.00 \pm 14.38$ & NS \\
\hline $\mathrm{HbA}_{1 \mathrm{c}}(\%)$ & $5.99 \pm 0.47$ & $6.04 \pm 0.47$ & $6.39 \pm 0.77^{* \dagger}$ & $6.21 \pm 0.55^{* \dagger}$ & $<0.001$ \\
\hline $\mathrm{HbA}_{1 \mathrm{c}}(\mathrm{mmol} / \mathrm{mol})$ & $42.0 \pm 5.1$ & $42.5 \pm 5.1$ & $46.3 \pm 8.4^{* \dagger}$ & $44.4 \pm 6.0^{* \dagger}$ & $<0.001$ \\
\hline Fasting glucose (mmol/l) & $5.24 \pm 0.67$ & $5.25 \pm 0.74$ & $6.01 \pm 1.22 *^{\dagger}$ & $5.65 \pm 0.80 *{ }^{\dagger}$ & $<0.001$ \\
\hline Fasting insulin (pmol/l) & $45.4 \pm 24.3$ & $55.5 \pm 29.8$ & $103.8 \pm 80.1^{* \dagger}$ & $108.7 \pm 56.8^{* \dagger}$ & $<0.001$ \\
\hline HIRI & $261 \pm 98$ & $311 \pm 94 * \$ \S$ & $710 \pm 419 * \dagger \S$ & $824 \pm 489 * \dagger$ & $<0.001$ \\
\hline MISI & $0.029 \pm 0.022$ & $0.005 \pm 0.003 * *$ & $0.027 \pm 0.022$ & $0.005 \pm 0.003 *$ & $<0.001$ \\
\hline ISI & $14.95 \pm 7.42$ & $10.51 \pm 4.50^{* \pm \S}$ & $6.55 \pm 2.85^{* \dagger \S}$ & $4.71 \pm 1.71 *+$ & $<0.001$ \\
\hline HOMA-IR & $1.90 \pm 0.75$ & $2.27 \pm 0.72 *$ & $5.22 \pm 3.14^{* \dagger}$ & $6.07 \pm 3.66^{* \dagger}$ & $<0.001$ \\
\hline IGI & $76.7 \pm 59.4$ & $120.1 \pm 108.2 * \star$ & $84.3 \pm 69.1$ & $129.7 \pm 90.8^{* \ddagger}$ & $<0.001$ \\
\hline Disposition index & $1,106 \pm 926$ & $1,176 \pm 1,104$ & $594 \pm 484^{* \dagger}$ & $678 \pm 489^{* \dagger}$ & $<0.001$ \\
\hline
\end{tabular}

Data are mean \pm SD unless indicated

${ }^{\mathrm{a}}$ Univariate model adjusted for age and sex, followed by Bonferroni's test

${ }^{*} p<0.05$ vs no IR

$\dagger p<0.05$ vs muscle IR

$\$ p<0.05$ vs liver IR

$\S p<0.05$ vs muscle+liver IR 
and liver IR groups presented higher HOMA-IR than muscle IR, and these three groups all had higher HOMA-IR compared with patients with no IR $(p<0.001)$. Finally, the disposition index was lower in patients with muscle+liver IR and liver IR than in those with muscle IR and no IR $(p<0.001)$.

\section{Comparative effect of the Mediterranean vs the low-fat} diet on variables of glycaemic control according to IR subgroups At baseline, there were no significant differences in the variables studied between the patients who were randomised to a low-fat diet or to a Mediterranean diet within each IR subgroup (Table 2).

HIRI significantly decreased after 2 years of follow-up with both dietary treatments in all subgroups $(p<0.001)$. However, patients with liver IR and muscle+liver IR presented a greater improvement in HIRI compared with those with no IR, measured as the mean percentage change. Specifically, HIRI decreased more in the low-fat and Mediterranean diet groups in patients with liver IR than in those with no IR $(-44.1 \%$ vs $-16.8 \%, p=0.002 ;-38.5 \%$ vs $-13.7 \%$, $p=0.017$, respectively). On the other hand, HIRI decreased more in patients with muscle+liver IR consuming the lowfat diet than in those with no IR consuming the low-fat diet $(-42.4 \%$ vs $-16.8 \%, p=0.012)$ (Table 2$)$. MISI significantly increased in the muscle IR and muscle+liver IR groups after 2 years of follow-up with both dietary treatments $(p<0.001$ and $p=0.003$, respectively), whereas it significantly decreased in the liver IR group with both dietary treatments $(p=0.001)$. In terms of mean percentage change, there were also significant differences between IR subgroups. Patients with muscle IR consuming the low-fat and Mediterranean diets presented a greater increase in MISI compared with those with no IR (172.9\% vs $-6.0 \%, p<0.001 ; 204.4 \%$ vs $-3.3 \%, p<0.001$, respectively) and with liver IR $(172.9 \%$ vs $-30.5 \%$, $p<0.001 ; 204.4 \%$ vs $-12.5 \%, p<0.001$, respectively). By contrast, MISI increased more in patients with muscle+liver IR consuming the low-fat diet than in those with no IR $(147.5 \%$ vs $-6.0 \%, p<0.001)$ and with liver IR $(147.5 \%$ vs $-30.5 \%, p<0.001$ ) (Table 2).

$\mathrm{HbA}_{1 \mathrm{c}}$ significantly decreased after 2 years of follow-up with both dietary treatments in all subgroups $(p<0.001)$, except in those with liver IR who consumed the Mediterranean diet (Table 2).

IGI decreased significantly from baseline to 2 years of follow-up in patients with muscle+liver IR $(p=0.015)$ consuming the low-fat diet. In terms of mean percentage change, there was a significant difference in the muscle IR group ( $p=0.044$ ) between those who consumed the low-fat diet (in whom IGI decreased) and those who consumed the Mediterranean diet (in whom IGI increased).

Finally, long-term consumption of the Mediterranean diet for 2 years led to an increase in disposition index that was only significant in muscle IR $(p=0.042)$ and muscle+liver IR patients $(p=0.048)$, whereas consumption of the low-fat diet led to an increase in disposition index that was only significant in liver IR patients $(p=0.017)$. Moreover, there were differences in the values of mean percentage change in the disposition index (Fig. 1). In muscle IR and muscle+liver IR patients, consumption of the Mediterranean diet led to a higher increase in disposition index compared with the low-fat diet $(55.0 \%$ vs $26.5 \%, p=0.020$ and $41.0 \%$ vs $14.8 \%, p=0.040$, respectively). By contrast, in liver IR patients the increase in disposition index was higher in those consuming the low-fat diet than in those consuming the Mediterranean diet $(67.3 \%$ vs $20.3 \%$, $p=0.014$ ).

ISI and HOMA-IR values significantly improved after 2 years of follow-up with both dietary treatments and in all subgroups (ESM Table 2).

Dietary intake and anthropometric measurements At baseline, there were no significant differences in Mediterranean diet score between the Mediterranean diet and the low-fat diet groups and between the IR subgroups (Table 3). After 2 years of follow-up, the Mediterranean diet score significantly decreased in patients following the low-fat diet but significantly increased in patients following the Mediterranean diet, in all subgroups. Accordingly, the change in mean percentage change in Mediterranean diet score was significantly greater in the Mediterranean diet group, for all the IR subgroups $(p<0.001)$.

As regards the anthropometric measurements, no IR, muscle IR and liver IR patients following the low-fat diet presented a decrease in weight and BMI $(p<0.05)$ (Table 3). No IR and liver IR patients consuming the low-fat diet also showed a significant decrease in waist circumference $(p<0.05)$. No changes were observed in anthropometric measurements in patients consuming the Mediterranean diet.

\section{Discussion}

This study provides new evidence that different dietary approaches (a Mediterranean diet or a low-fat diet) may provide extra benefits in improving beta cell function in patients with CHD, depending on the presence or absence of muscle and/or liver IR. Our findings suggest that, although both diets improve insulin sensitivity, there are some differences based on basal IR phenotypes. MISI increased only in patients who presented muscle IR at baseline (alone or combined with liver IR), whereas HIRI presented a greater decrease in patients with baseline liver IR relative to the other groups. Moreover, according to IGI and disposition index data, a low-fat diet might be of more benefit to patients with liver IR, whereas a Mediterranean diet might be more beneficial to patients with muscle IR and muscle+liver IR.

There is compelling clinical trial evidence to show that the most cost-effective method to prevent type 2 diabetes is 


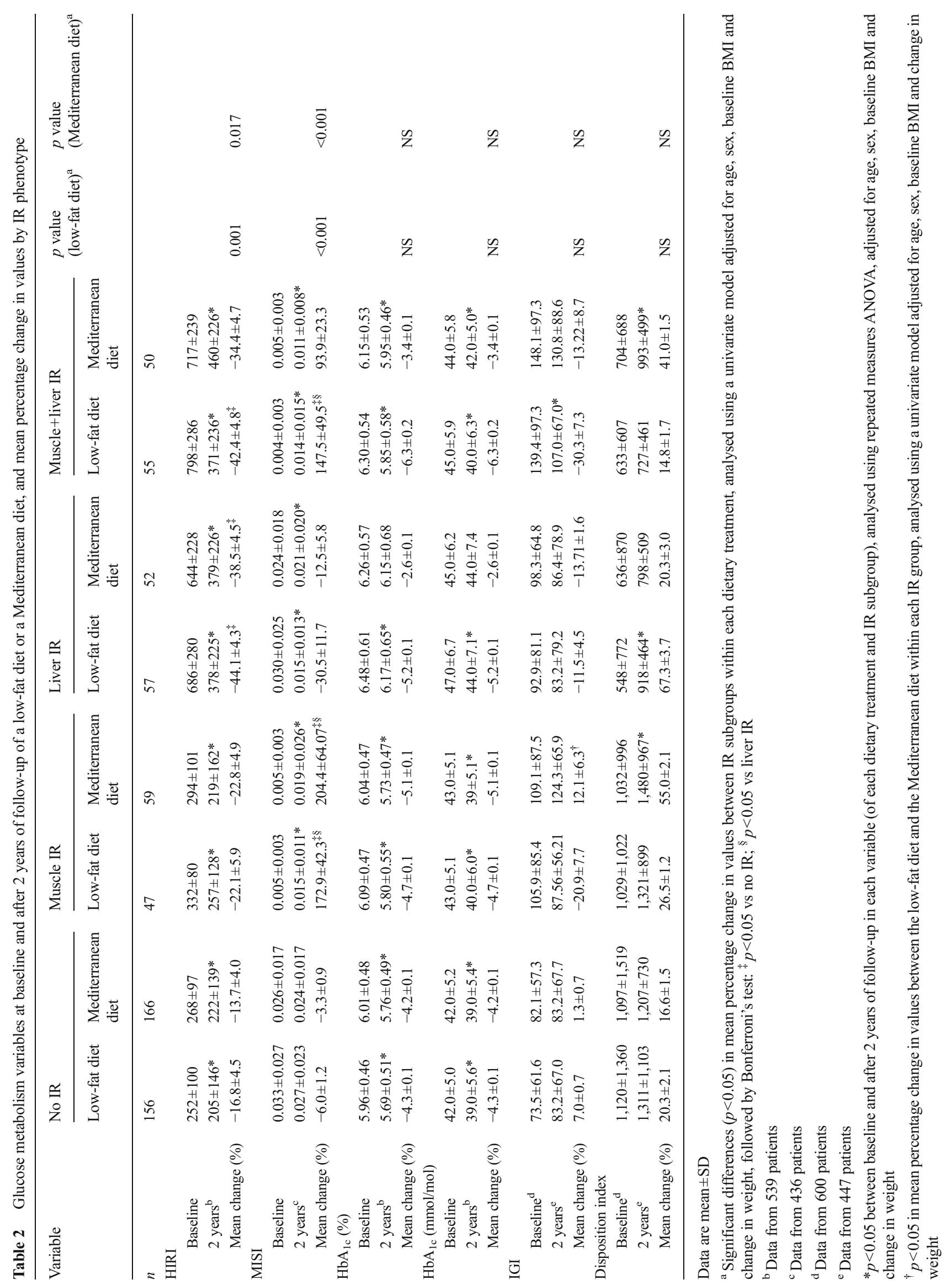




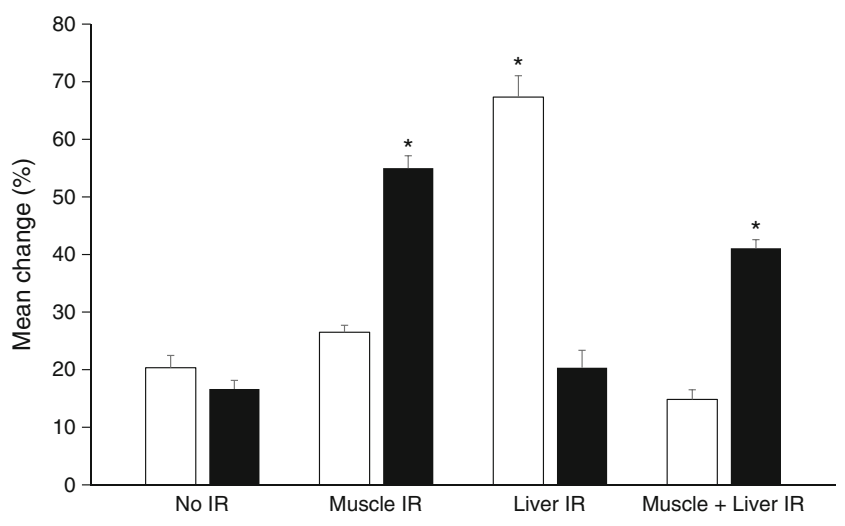

Fig. 1 Mean percentage change in values of disposition index between baseline and after 2 years of follow-up by IR phenotype. ${ }^{*} p<0.05$ between low-fat diet (white bars) and Mediterranean diet (black bars) in each IR subgroup analysed using a univariate model adjusted for age, sex, baseline BMI and change in weight

lifestyle intervention, which includes changes in exercise and dietary habits [22-24]. In several of these long-term intervention studies, the main dietary goals in the intervention groups were a low-fat, calorie-restricted diet with a low content of saturated fatty acids and a higher dietary fibre intake. As a consequence, participants in the active treatment groups achieved significant weight loss, which appeared to be the main factor in reducing incident diabetes compared with control groups. Other prospective studies have demonstrated that greater adherence to a Mediterranean diet is associated with a significant reduction in the risk of diabetes $[25,26]$. In this context, the Effects of the Mediterranean Diet on the Primary Prevention of Cardiovascular Diseases (PREDIMED) trial performed a long-term intervention, with a median followup of 4.1 years, in non-diabetic individuals comparing three dietary interventions: a Mediterranean diet supplemented with extra-virgin olive oil; a Mediterranean diet supplemented with mixed nuts; and a control diet consisting of advice to reduce intake of all types of fat. The authors concluded that a Mediterranean diet enriched with extra-virgin olive oil but without energy restrictions reduced type 2 diabetes incidence compared with the other two diets [27]. Despite the success of these studies, two recent reviews highlighted the need to

Table 3 Mediterranean diet adherence score and anthropometric measurements at baseline and after 2 years of follow-up of a low-fat diet or a Mediterranean diet, and mean percentage change in values by IR phenotype

\begin{tabular}{|c|c|c|c|c|c|c|c|c|}
\hline \multirow[t]{2}{*}{ Variable } & \multicolumn{2}{|l|}{ No IR } & \multicolumn{2}{|l|}{ Muscle IR } & \multicolumn{2}{|l|}{ Liver IR } & \multicolumn{2}{|c|}{ Muscle+liver IR } \\
\hline & Low-fat diet & $\begin{array}{l}\text { Mediterranean } \\
\text { diet }\end{array}$ & Low-fat diet & $\begin{array}{l}\text { Mediterranean } \\
\text { diet }\end{array}$ & Low-fat diet & $\begin{array}{l}\text { Mediterranean } \\
\text { diet }\end{array}$ & Low-fat diet & $\begin{array}{l}\text { Mediterranean } \\
\text { diet }\end{array}$ \\
\hline \multicolumn{9}{|c|}{ Mediterranean diet score } \\
\hline Baseline & $8.67 \pm 2.06$ & $8.96 \pm 2.00$ & $8.79 \pm 1.84$ & $8.95 \pm 2.37$ & $8.50 \pm 1.99$ & $8.68 \pm 1.96$ & $8.35 \pm 1.79$ & $8.92 \pm 2.02$ \\
\hline 2 years & $7.57 \pm 1.68^{*}$ & $11.62 \pm 1.71 *$ & $7.53 \pm 1.71^{*}$ & $11.45 \pm 1.79 *$ & $7.40 \pm 1.65^{*}$ & $11.36 \pm 2.03^{*}$ & $7.60 \pm 1.51^{*}$ & $12.00 \pm 1.29 *$ \\
\hline $\begin{array}{l}\text { Mean change } \\
(\%)\end{array}$ & $-9.3 \pm 2.6$ & $38.2 \pm 4.5^{\dagger}$ & $-11.4 \pm 2.4$ & $36 \pm 5.7^{\dagger}$ & $-11.5 \pm 2.2$ & $41.0 \pm 7.2^{\dagger}$ & $-5.9 \pm 3.1$ & $40.7 \pm 4.0^{\dagger}$ \\
\hline \multicolumn{9}{|l|}{ Weight $(\mathrm{kg})$} \\
\hline Baseline & $79.93 \pm 12.49$ & $80.72 \pm 12.02$ & $80.43 \pm 9.94$ & $78.04 \pm 13.46$ & $91.20 \pm 14.81$ & $91.87 \pm 13.23$ & $88.86 \pm 15.23$ & $90.09 \pm 13.04$ \\
\hline 2 years & $78.57 \pm 12.47^{*}$ & $79.64 \pm 12.11$ & $78.72 \pm 10.30^{*}$ & $79.30 \pm 14.49$ & $90.22 \pm 13.32 *$ & $90.38 \pm 14.15$ & $87.34 \pm 17.74$ & $91.31 \pm 15.12$ \\
\hline $\begin{array}{l}\text { Mean change } \\
(\%)\end{array}$ & $-2.4 \pm 0.5$ & $-1.7 \pm 0.6$ & $-1.9 \pm 0.6$ & $0.1 \pm 1.1$ & $-2.2 \pm 0.4$ & $-0.8 \pm 0.7$ & $-0.9 \pm 0.5$ & $0.4 \pm 1.4$ \\
\hline \multicolumn{9}{|l|}{ BMI $\left(\mathrm{kg} / \mathrm{m}^{2}\right)$} \\
\hline Baseline & $29.08 \pm 3.90$ & $29.49 \pm 3.99$ & $30.08 \pm 3.24$ & $29.26 \pm 3.95$ & $32.35 \pm 4.77$ & $32.20 \pm 3.67$ & $32.73 \pm 5.07$ & $32.59 \pm 4.32$ \\
\hline 2 years & $28.56 \pm 4.04^{*}$ & $29.21 \pm 4.32$ & $29.25 \pm 3.24^{*}$ & $29.69 \pm 4.70$ & $31.97 \pm 4.48^{*}$ & $31.85 \pm 3.87$ & $32.04 \pm 5.46$ & $33.02 \pm 4.84$ \\
\hline $\begin{array}{l}\text { Mean change } \\
(\%)\end{array}$ & $-2.2 \pm 0.5$ & $-1.2 \pm 0.4$ & $-1.7 \pm 0.4$ & $0.5 \pm 0.8$ & $-1.9 \pm 0.7$ & $-0.6 \pm 0.9$ & $-1.0 \pm 0.5$ & $0.9 \pm 1.2$ \\
\hline \multicolumn{9}{|c|}{ Waist circumference $(\mathrm{cm})$} \\
\hline Baseline & $100.2 \pm 10.1$ & $100.6 \pm 10.1$ & $101.1 \pm 8.2$ & $99.6 \pm 11.8$ & $109.6 \pm 10.9$ & $109.0 \pm 7.9$ & $108.5 \pm 11.5$ & $107.4 \pm 10.7$ \\
\hline 2 years & $98.4 \pm 10.3^{*}$ & $99.3 \pm 10.0$ & $100.5 \pm 8.4$ & $100.1 \pm 11.9$ & $108.0 \pm 10.4^{*}$ & $107.4 \pm 8.8$ & $107.9 \pm 11.8$ & $107.9 \pm 11.6$ \\
\hline $\begin{array}{l}\text { Mean change } \\
(\%)\end{array}$ & $-2.2 \pm 0.7$ & $-1.4 \pm 0.6$ & $-1.5 \pm 0.6$ & $-1.7 \pm 0.6$ & $-1.7 \pm 0.5$ & $-2.0 \pm 0.5$ & $-0.8 \pm 0.6$ & $0.6 \pm 0.8$ \\
\hline
\end{tabular}

\section{Data are mean \pm SD}

There were no significant differences between the IR groups within each dietary treatment (the low-fat diet or the Mediterranean diet) for all the variables ${ }^{*} p<0.05$ between baseline and after 2 years of follow-up in each variable (of each dietary treatment and IR subgroup), analysed using repeated measures ANOVA, adjusted for age, sex, baseline BMI and change in weight

${ }^{\dagger} p<0.05$ in mean percentage change in values between the low-fat diet and the Mediterranean diet within each IR group, analysed using a univariate model adjusted for age, sex, baseline BMI and change in weight 
perform intervention trials in order to determine specific dietary patterns to offer recommendations to vulnerable individuals $[5,26]$. The approach of the present study goes further, since it also takes into account the fact that the different pathophysiology of the distinct prediabetic states (muscle IR, liver IR and muscle+liver IR) might provide a basis for the development of individualised prevention of type 2 diabetes [28]. Therefore, we consider that our findings may have important clinical and public health applications, especially in the field of personalised medicine.

The diets studied in the intervention (Mediterranean and low-fat diets) are considered healthy diets [5]: both are rich in whole grain foods, fruit and vegetables, with a moderate consumption of fish and low-fat dairy products, and are low in saturated fat, meat and simple sugars. We observed that levels of $\mathrm{HbA}_{1 \mathrm{c}}$ were significantly reduced, which indicates that both diets improve glycaemic control [29] and is in agreement with results found in several intervention studies [30, 31]. We also found that both diets improved insulin sensitivity, although with differences according to tissue-specific IR. HIRI decreased in all patients with both diets, although this improvement seemed to be greater in liver IR and muscle+liver IR patients. By contrast, MISI was increased only in patients with muscle IR or muscle+liver IR after 2 years of follow-up of both diets. That is relevant, since the disposition index takes into account insulin secretion and insulin sensitivity, and the calculation itself is affected by tissue-specific IR [32].

Low-fat diets, with a content of $<30 \%$ of energy from total fat, are considered the best suited for type 2 diabetes prevention, according to many scientific societies, including the ADA and the Diabetes and Nutrition Study Group of the European Association for the Study of Diabetes [8, 33]. According to our results, this recommendation may be especially encouraged in patients with liver IR, whose disposition index was increased on the low-fat diet compared with those on the Mediterranean diet. It is important to note that, although neither diet in this study was calorie-restricted, the weight, BMI and waist circumference of the patients on the low-fat diet significantly decreased during follow-up, especially in those in the liver IR group. Our results agree with those of other intervention studies, which related weight loss to reduced diabetes incidence, even when the difference in weight was minimal [34]. In addition, it has been shown that a relatively small decrease in BMI is associated with a considerable reduction in hepatic triacylglycerol content, which might be related to a reduction in the severity of IR [35]. Therefore, this weight loss could diminish hepatic triacylglycerol content and IR, and thus enhance gluconeogenesis and favour beta cell function in our liver IR patients.

Recent research supports the idea that a traditional Mediterranean dietary pattern, even without energy restriction, can also help in type 2 diabetes prevention [27, 36]. The main differences between the low-fat and the
Mediterranean diets, which were reflected in the adherence questionnaire, were found in certain unique characteristics of the Mediterranean diet, such as the moderate consumption of wine and nuts and the widespread use of extra-virgin olive oil, which means that it is considered a highunsaturated fat diet $(>35 \%$ of energy from fat, of which $22 \%$ were monounsaturated fatty acids). We found a specific extra benefit of consuming the Mediterranean diet in patients with muscle IR, who presented a significant increase in IGI and disposition index compared with patients who consumed the low-fat diet; this was the case in muscle+liver IR patients, who also presented an increase in disposition index. This could be linked to the fact suggested in previous works that a diet high in monounsaturated fatty acids downregulates genes involved in lipogenesis and tends to decrease muscle triacylglycerol and diacylglycerol levels in skeletal muscle from insulinresistant individuals [37]. This may be supported by the improvement in insulin sensitivity and beta cell function that we observed in our muscle IR and muscle+liver IR patients. Moreover, it has been reported that extra-virgin olive oil consumption, compared with a low-fat meal, enhances the glucagon-like peptide-1 response [31]. This hormone has been shown to suppress endogenous glucose production potentially via effects on insulin pulsatility, but there is also some evidence that it promotes peripheral glucose uptake mostly related to neural mechanisms [38].

Some limitations of the current study must be mentioned. Type 2 diabetes prevention was not the primary endpoint of the CORDIOPREV trial, but was a secondary analysis conducted in the subgroup of cardiovascular patients not treated with glucose-lowering drugs at baseline (CORDIOPREVDIAB study). However, there are no reasons to believe that the randomisation would not have worked in such a large subset of participants. Another point is that the study sample consisted of older white patients with CHD, which limits the generalisation of our results to clinically healthy individuals and to other age groups or ethnicities. Finally, we experienced greater losses during follow-up in the low-fat diet group, although this seems not to have affected the results after 2 years of follow-up.

In spite of these limitations, to the best of our knowledge this study is the first long-term intervention comparing the effects of two dietary patterns (Mediterranean diet or low-fat diet) on tissue-specific IR and beta cell function in cardiovascular patients not treated for diabetes, according to muscle and/or liver IR. Our findings suggest that, although both diets improve insulin sensitivity, there are differences based on basal IR phenotypes. Moreover, according to IGI and disposition index data, the low-fat diet might be more beneficial to patients with liver IR, whereas the patients with skeletal muscle IR and those with both liver and muscle IR might benefit more from a Mediterranean diet. Although further 
studies are needed to confirm these results in other population groups, such as in healthy individuals at risk of progression to diabetes and in those with different ethnicity, these findings may guide the implementation of personalised dietary interventions tailored to the different states of IR, thus achieving more efficient prevention of type 2 diabetes in vulnerable patients.

Acknowledgements The authors would like to thank to Fundacion Patrimonio Comunal Olivarero and the Escuela Andaluza de Salud Publica (EASP), Granada, Spain, which carried out the randomisation process of this study; and also the volunteers who participated in the study.

Funding The present study was supported by the Ministerio de Economia y Competitividad (AGL2012/39615 to JL-M) and by the Ministerio de Ciencia e Innovacion (PIE14/00005 to JL-M and PI13/00023 to JD-L). It was also partly supported by a research grant from the European Commission (NUTRITECH European Integrated Project-289511). RB-R is supported by an ISCIII postdoctoral research contract (Sara Borrell) and JFA-D is supported by an Instituto de Salud Carlos III research contract (Programa Rio-Hortega).

Duality of interest The authors declare that there is no duality of interest associated with this manuscript.

Contribution statement JL-M, JD-L and FP-J conceived and designed the study; RB-R, JFA-D, GMQ-N, JD-L, PP-M and CM performed the experiments and participated in the acquisition of data; RB-R, JFA-D, $\mathrm{SW}, \mathrm{BvO}, \mathrm{JMO}$ and JL-M analysed and interpreted the data; RB-R and JL-M drafted the manuscript; JD-L, PP-M, JMO and BvO provided critical revision of the paper for the main intellectual content. All authors contributed substantially to aspects of study design or acquisition of data, to drafting of the article, or to critical revision for important intellectual content. All authors have given their final approval of the version to be published. JL-M is the guarantor of this work.

\section{References}

1. World Health Organization (2008) 2008-2013 action plan for the global strategy for the prevention and control of noncommunicable diseases: prevent and control cardiovascular diseases, cancers, chronic respiratory diseases and diabetes. WHO, Geneva

2. DeFronzo RA (2010) Insulin resistance, lipotoxicity, type 2 diabetes and atherosclerosis: the missing links. The Claude Bernard Lecture 2009. Diabetologia 53:1270-1287

3. Matthaei S, Stumvoll M, Kellerer M, Haring HU (2000) Pathophysiology and pharmacological treatment of insulin resistance. Endocr Rev 21:585-618

4. Weir GC, Bonner-Weir S (2004) Five stages of evolving beta-cell dysfunction during progression to diabetes. Diabetes 53(Suppl 3): S16-S21

5. Salas-Salvado J, Martinez-Gonzalez MA, Bullo M, Ros E (2011) The role of diet in the prevention of type 2 diabetes. Nutr Metab Cardiovasc Dis 21(Suppl 2):B32-B48

6. Lopez-Miranda J, Perez-Jimenez F, Ros E et al (2010) Olive oil and health: summary of the II international conference on olive oil and health consensus report, Jaen and Cordoba (Spain) 2008. Nutr Metab Cardiovasc Dis 20:284-294

7. Salas-Salvado J, Bullo M, Babio N et al (2011) Reduction in the incidence of type 2 diabetes with the Mediterranean diet: results of the PREDIMED-Reus nutrition intervention randomized trial. Diabetes Care 34:14-19

8. American Diabetes Association (2014) Standards of medical care in diabetes-2014. Diabetes Care 37(Suppl 1):S14-S80

9. Goodpaster BH, Coen PM (2012) Do acute exercise and diet reveal the molecular basis for metabolic flexibility in skeletal muscle? Diabetes 61:983

10. Tiikkainen M, Hakkinen AM, Korsheninnikova E, Nyman T, Makimattila S, Yki-Järvinen H (2004) Effects of rosiglitazone and metformin on liver fat content, hepatic insulin resistance, insulin clearance, and gene expression in adipose tissue in patients with type 2 diabetes. Diabetes 53:2169-2176

11. Garcia-Rios A, Gomez-Delgado FJ, Garaulet M et al (2014) Beneficial effect of CLOCK gene polymorphism rs1801260 in combination with low-fat diet on insulin metabolism in the patients with metabolic syndrome. Chronobiol Int 31:401-408

12. Perez-Martinez P, Alcala-Diaz JF, Delgado-Lista J et al (2014) Metabolic phenotypes of obesity influence triglyceride and inflammation homoeostasis. Eur J Clin Invest 44:1053-1064

13. Portaluppi F, Smolensky MH, Touitou Y (2010) Ethics and methods for biological rhythm research on animals and human beings. Chronobiol Int 27:1911-1929

14. Fernandez-Ballart JD, Pinol JL, Zazpe I et al (2010) Relative validity of a semi-quantitative food-frequency questionnaire in an elderly Mediterranean population of Spain. Br J Nutr 103:1808-1816

15. Estruch R, Ros E, Salas-Salvadó J et al (2013) Primary prevention of cardiovascular disease with a Mediterranean diet. N Engl J Med 368:1279-1290

16. Matsuda M, DeFronzo RA (1999) Insulin sensitivity indices obtained from oral glucose tolerance testing: comparison with the euglycemic insulin clamp. Diabetes Care 22:1462-1470

17. Abdul-Ghani MA, Matsuda M, Balas B, DeFronzo RA (2007) Muscle and liver insulin resistance indexes derived from the oral glucose tolerance test. Diabetes Care 30:89-94

18. Song Y, Manson JE, Tinker L et al (2007) Insulin sensitivity and insulin secretion determined by homeostasis model assessment and risk of diabetes in a multiethnic cohort of women: the Women's Health Initiative Observational Study. Diabetes Care 30:1747-1752

19. Hanson RL, Pratley RE, Bogardus C et al (2000) Evaluation of simple indices of insulin sensitivity and insulin secretion for use in epidemiologic studies. Am J Epidemiol 151:190-198

20. Tang W, Fu Q, Zhang Q et al (2014) The association between serum uric acid and residual beta -cell function in type 2 diabetes. $\mathrm{J}$ Diabetes Res 2014:709691

21. Abdul-Ghani MA, Matsuda M, DeFronzo RA (2008) Strong association between insulin resistance in liver and skeletal muscle in non-diabetic subjects. Diabet Med 25:1289-1294

22. Pan XR, Li GW, Hu YH et al (1997) Effects of diet and exercise in preventing NIDDM in people with impaired glucose tolerance. The Da Qing IGT and Diabetes Study. Diabetes Care 20:537-544

23. Tuomilehto J, Lindstrom J, Eriksson JG et al (2001) Prevention of type 2 diabetes mellitus by changes in lifestyle among subjects with impaired glucose tolerance. N Engl J Med 344:1343-1350

24. Knowler WC, Barrett-Connor E, Fowler SE et al (2002) Reduction in the incidence of type 2 diabetes with lifestyle intervention or metformin. N Engl J Med 346:393-403

25. Schwingshackl L, Missbach B, Konig J, Hoffmann G (2014) Adherence to a Mediterranean diet and risk of diabetes: a systematic review and meta-analysis. Public Health Nutr: 1-8

26. Esposito K, Chiodini P, Maiorino MI, Bellastella G, Panagiotakos D, Giugliano D (2014) Which diet for prevention of type 2 diabetes? A meta-analysis of prospective studies. Endocrine 47:107-116

27. Salas-Salvado J, Bullo M, Estruch R et al (2014) Prevention of diabetes with Mediterranean diets: a subgroup analysis of a randomized trial. Ann Intern Med 160:1-10 
28. Faerch K, Borch-Johnsen K, Holst JJ, Vaag A (2009) Pathophysiology and aetiology of impaired fasting glycaemia and impaired glucose tolerance: does it matter for prevention and treatment of type 2 diabetes? Diabetologia 52:1714-1723

29. Jenkins DJ, Kendall CW, Banach MS et al (2011) Nuts as a replacement for carbohydrates in the diabetic diet. Diabetes Care 34:17061711

30. Sleiman D, Al-Badri MR, Azar ST (2015) Effect of Mediterranean diet in diabetes control and cardiovascular risk modification: a systematic review. Front Public Health 3:69

31. Paniagua JA, de la Sacristana AG, Sanchez E et al (2007) A MUFA-rich diet improves postprandial glucose, lipid and GLP-1 responses in insulin-resistant subjects. J Am Coll Nutr 26:434-444

32. Faerch K, Brons C, Alibegovic AC, Vaag A (2010) The disposition index: adjustment for peripheral vs hepatic insulin sensitivity? J Physiol 588:759-764
33. Mann JI, De Leeuw I, Hermansen K et al (2004) Evidence-based nutritional approaches to the treatment and prevention of diabetes mellitus. Nutr Metab Cardiovasc Dis 14:373-394

34. Hamman RF, Wing RR, Edelstein SL et al (2006) Effect of weight loss with lifestyle intervention on risk of diabetes. Diabetes Care 29: 2102-2107

35. Snel M, Jonker JT, Schoones J et al (2012) Ectopic fat and insulin resistance: pathophysiology and effect of diet and lifestyle interventions. Int J Endocrinol 2012:983814

36. Riserus U, Willett WC, Hu FB (2009) Dietary fats and prevention of type 2 diabetes. Prog Lipid Res 48:44-51

37. Jans A, van Hees AM, Gjelstad IM et al (2012) Impact of dietary fat quantity and quality on skeletal muscle fatty acid metabolism in subjects with the metabolic syndrome. Metabolism 61:1554-1565

38. Sandoval DA, D'Alessio DA (2015) Physiology of proglucagon peptides: role of glucagon and GLP-1 in health and disease. Physiol Rev 95:513-548 